TEI

JOURNAL OF THE
Journal of the Text Encoding Initiative

Issue 2 | February 2012

Selected Papers from the 2010 TEI Conference

\title{
Editorial Introduction to the Second Issue
}

\section{Christian Wittern}

\section{(2) OpenEdition}

\section{Journals}

Electronic version

URL: http://journals.openedition.org/jtei/369

DOI: $10.4000 /$ jtei.369

ISSN: 2162-5603

\section{Publisher}

TEl Consortium

\section{Electronic reference}

Christian Wittern, «Editorial Introduction to the Second Issue », Journal of the Text Encoding Initiative [Online], Issue 2 | February 2012, Online since 03 February 2012, connection on 21 April 2019. URL : http://journals.openedition.org/jtei/369; DOI : 10.4000/jtei.369

This text was automatically generated on 21 April 2019

TEI Consortium 2012 (Creative Commons Attribution-NoDerivs 3.0 Unported License) 


\title{
Editorial Introduction to the Second Issue
}

\author{
Christian Wittern
}

1 It is my pleasure to introduce this second issue of the Journal of the Text Encoding Initiative, containing selected papers from the 2010 TEI Members Meeting, which was held from November 11 to 13 at the University of Zadar in Croatia.

2 All the papers in this volume are derived from presentations at the conference. The theme of the conference was Digital Texts and Language Resources, with a rather open scope of possible applications. The submitted presentations and also the papers included in this volume clearly show a focus on themes which seem to be important to the TEI community, namely using the TEI for the encoding of manuscripts and manuscript descriptions, and the metadata used within the TEI and the relation to other metadata standards. A third, related topic, which is underlying in some of the papers and is foregrounded in others, is the question of tools for use with the TEI encoding schema.

In their contribution "Mirroring the Community: The Present and Future of the TEI Community for Manuscript Encoding," Marjorie Burghart and Malte Rehbein report on the results of a survey among users of the TEI for the encoding of manuscripts. This survey has been conducted to gauge difficulties encountered by the users and provide guidance for future developments. One of the conclusions they draw from the survey is that a steep learning curve and many obstacles in applying the TEI to manuscripts have to be overcome in order to successfully work with the TEI. The TEI community needs to address these problems by providing more examples, best-practice guidelines and howto's.

4 In contrast to this broad survey, Cristina Vertan and Stefanie Reimers focus on a more specific aspect of dealing with the TEI encoding scheme, by reporting on a "A TEI-based Application for Editing Manuscript Descriptions." This is an attempt to allow codicological and paleographical specialists to create manuscript descriptions compatible with both the TEI module for manuscript descriptions and the DFG guidelines without needing to delve into the technical aspects of this work using a form based data entry 
interface. This work draws also on experience of the ENRICH project but with a focus on the need of classicists.

The third contribution, by Mohammed Ourabah Soualah and Mohamed Hassoun, "A TEI P5 Manuscript Description Adaptation for Cataloguing Digitized Arabic Manuscripts," also concerns itself with manuscript descriptions, but with manuscripts in Arabic in particular. It is not surprising that there are special problems with this material, and this report on a real world application of the TEI to works in a non-Western language is especially welcome. Problems encountered and solved fall in three categories: language representation, representation of specific name types used in the Arabic cultural sphere, and specifics of the writing style; however, the paper also shows the adaptability of the TEI scheme to such requirements.

6 Now turning to metadata in a more general sense, Grant Leyton Simpson and Dot Porter ask themselves what happens if the usual direction of transforming TEI to HTML (and related formats) for publication is reversed. In their contribution "Transforming Backward: HTML and HTML+RDFa to TEI," they look into a workflow that takes HTML as its input and up-converts this to valid TEI markup. This has the potential to be used in legacy data for the conversion to an archival format, but as is shown in the paper, it is difficult to generalize since it depends very much on the specific use of certain tags, and needs manual checking.

7 Gerhard Budin, Heinrich Kabas, and Karlheinz Moerth continue with a contribution focusing on metadata with "Towards Finer Granularity in Metadata: Analysing the Contents of Retro-Digitised Periodicals." The aim is to increase and improve the quality of metadata in the corpus of the Austrian Academy of Sciences, which holds a wide variety of different materials with a diversity of markup density and usage. With an improved workflow and better tools, they show how findability in the corpus can be significantly improved. The tools generated in this project are available from the project website.

In a similar vein, Neven Jovanovic tries to enhance a collection of Latin texts for use by a larger scholarly community, as described in his contribution "CroALa: Enhancing a TEIencoded Text Collection." Jovanovic is not only concerned with fine-tuning the metadata but also with integrating the texts with other tools as a way to enhance the overall usability of the collection, either by relying on client-side tools that can be installed into a web browser or by connecting to other sites through web services. This is an interesting example of how advanced digital humanists can, without full training in programming, tweak the tools to their purpose by mashing and mixing what is available. It also shows that publication in a variety of formats and contexts increases the visibility within the community and thus, ultimately the usability of the materials.

With this contribution, the focus has already shifted in some way to the tools for publication, rather than simply on the metadata; this serves as a transition to the seventh contribution, in which Sebastian Pape, Christof Schöch, and Lutz Wegner focus entirely on tools for the publication of TEI texts in their contribution "TEICHI and the Tools Paradox: Developing a Publishing Framework for Digital Editions." They introduce a new framework for an easy-to-use framework for the publication of TEI documents and discuss it by comparing it to two other publication frameworks, namely the Versioning Machine and Scalable Architecture for Digital Editions (SADE).

10 Taken together, the articles in this issue represent the wide variety of international and multi-lingual uses of the TEI that were a feature of the 2010 conference, while building on 
and expanding the range of themes, research areas, and activities explored in the first issue.

AUTHOR

\section{CHRISTIAN WITTERN}

Christian Wittern is Associate Professor at the Center for Informatics in East Asian Studies, Kyoto University, Japan 